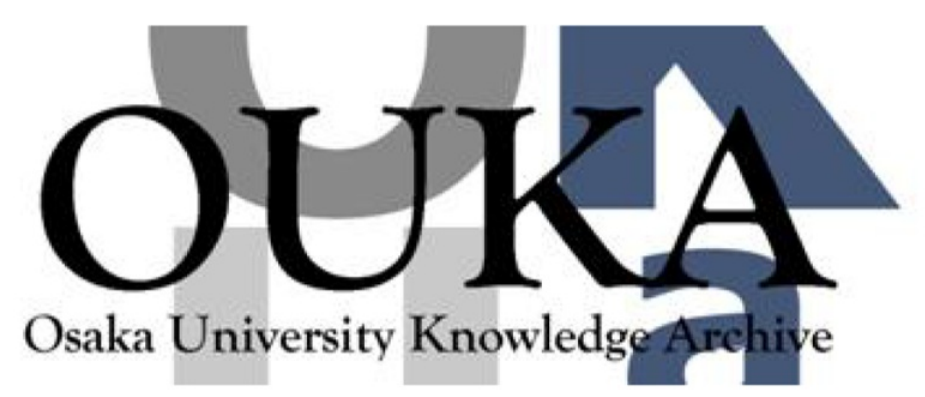

\begin{tabular}{|c|c|}
\hline Title & $\begin{array}{l}\text { Molecular dynamics evaluation of self-diffusion } \\
\text { in Yukawa systems }\end{array}$ \\
\hline Author (s) & Ohta, H.; Hamaguchi, S. \\
\hline Citation & Physics of Plasmas. 7(11) p. 4506-p. 4514 \\
\hline Issue Date & $2000-11$ \\
\hline oaire:version & VoR \\
\hline URL & https://hdl. handle. net/11094/78496 \\
\hline rights & $\begin{array}{l}\text { This article may be downloaded for personal use } \\
\text { only. Any other use requires prior permission } \\
\text { of the author and AIP Publishing. This article } \\
\text { appeared in Physics of Plasmas } 7,4506 \text { (2000) } \\
\text { and may be found at } \\
\text { https://doi.org/10.1063/1.1316084. }\end{array}$ \\
\hline Note & \\
\hline
\end{tabular}

Osaka University Knowledge Archive : OUKA

https://ir. Library. osaka-u. ac. jp/

0saka University 


\section{Molecular dynamics evaluation of self- diffusion in Yukawa systems}

Cite as: Physics of Plasmas 7, 4506 (2000); https://doi.org/10.1063/1.1316084

Submitted: 30 May 2000 . Accepted: 15 August 2000 . Published Online: 19 October 2000

H. Ohta, and S. Hamaguchi

\section{ARTICLES YOU MAY BE INTERESTED IN}

Shear viscosity of strongly coupled Yukawa systems

Physics of Plasmas 9, 1210 (2002); https://doi.org/10.1063/1.1459708

Phase diagram and dynamics of Yukawa systems

The Journal of Chemical Physics 88, 3286 (1988); https://doi.org/10.1063/1.453924

Phase diagram of Yukawa systems near the one-component-plasma limit revisited

The Journal of Chemical Physics 105, 7641 (1996); https://doi.org/10.1063/1.472802

\section{AIP Advances Fluids and Plasmas Collection}




\title{
Molecular dynamics evaluation of self-diffusion in Yukawa systems
}

\author{
H. Ohta and S. Hamaguchi \\ Department of Fundamental Energy Science, Kyoto University, Gokasho, Uji, Kyoto 611-0011, Japan
}

(Received 30 May 2000; accepted 15 August 2000)

\begin{abstract}
Self-diffusion coefficients of Yukawa systems in the fluid phase are obtained from molecular dynamics simulations in a wide range of the thermodynamical parameters. The Yukawa system is a collection of particles interacting through Yukawa (i.e., screened Coulomb) potentials, which may serve as a model for charged dust particles in a plasma or colloidal particles in electrolytes. The self-diffusion coefficients are found to follow a simple scaling law with respect to the system temperature, which is consistent with the universal scaling (i.e., temperature scaling independent of the ratio of interparticle distance to screening length) observed by Robbins et al. [J. Chem. Phys. 88, 3286 (1988)] if the fluid system is near solidification. Also discussed is the velocity autocorrelation function, which is in part used to determine the self-diffusion coefficients through the Green-Kubo formula. (C) 2000 American Institute of Physics. [S1070-664X(00)04811-4]
\end{abstract}

\section{INTRODUCTION}

The Yukawa system is a collection of particles interacting through Yukawa (i.e., screened Coulomb) pair potentials

$$
\phi(r)=\frac{Q^{2}}{4 \pi \epsilon_{0}} \frac{\exp \left(-k_{D} r\right)}{r} .
$$

Here $r$ is the distance between two Yukawa particles and $k_{D}^{-1}$ is the screening length. Yukawa systems can serve as a model for charged dust particles (particulates) immersed in plasmas $^{1-6}$ - dusty plasmas-or colloidal particles suspended in electrolytes, ${ }^{7-9}$ where each particle has electric charge $Q$ and the electric field potential around each particle is screened with the screening length $k_{D}^{-1}$. In the case of dusty plasmas, particulates are typically charged negatively due to the high mobility of electrons and the screening arises from the formation of a sheath around each particulate. In laboratory plasmas, dusty plasmas are often formed in glow discharges with mesoscopic particles (the sizes and electrical charges of which are of order $1 \mu \mathrm{m}$ and a few thousand electron charges). Recent laboratory experiments ${ }^{10}$ have shown that the interparticle potential of charged dust particles in a plasma is indeed given by the Yukawa potential with high accuracy in the absence of plasma flows. Of course, in actual dusty plasmas, dynamics of charged dust particles can be more complex and subject to several other forces, such as the collision with background neutral gases. For example, ion flows passing around a charged dust particle are known to create a wake field behind the particle, resulting in anisotropic attractive forces among charged particles. ${ }^{11,12}$ The Yukawa model therefore may be used as a simplified model for charged dust particles in a plasma, on which one can construct more realistic models to represent actual dusty plasmas under various conditions.

The Yukawa system may also be of special interest as a mathematical model for many-body systems since it allows the full range of behavior between systems governed by short-range and long-range forces. For example, in the limit of no screening (i.e., $k_{D}=0$ ), the system is known as the one-component plasma (OCP) ${ }^{13-17}$ which represents a system of ions when electrons are extremely mobile. The OCP has often been used as a classical model of the dense interiors of white dwarfs, where ions are freely interact with each other through Coulomb potentials in degenerate electron backgrounds. As the screening increases (i.e., $k_{D}$ increases), the system acquires more characteristics of charge neutral fluids.

The Yukawa system in thermodynamical equilibrium can be characterized by two dimensionless parameters: $\kappa$ $=k_{D} a$, i.e., the ratio of the interparticle distance $a$ $=(3 / 4 \pi n)^{1 / 3}$ (where $n$ is the particle number density) to the screening length $k_{D}^{-1}$ and $\Gamma=Q^{2} / 4 \pi \epsilon_{0} a T$, i.e., the inverse of the system temperature (thermal energy) $T$ measured in units of $Q^{2} / 4 \pi \epsilon_{0} a$. The system is called "strongly coupled" if the coupling parameter $\Gamma^{*}=\Gamma \exp (-\kappa)$, i.e., the ratio of the average interparticle potential energy to the average kinetic energy, is comparable with or greater than unity. In particular, if the system is sufficiently cooled, i.e., the total kinetic energy becomes sufficiently small compared with the total potential energy (i.e., internal energy) the system can undergo phase transition from the fluid phase to the solid phase. We denote the critical $\Gamma$ by $\Gamma_{m}$, where the subscript $m$ represents "melting." Table I lists the values of $\Gamma_{m}$ that we used in our data analyses in this paper. These values are taken from Table X of Ref. 3 and the fitting formula ${ }^{18}$ given by Eq. (21) of Ref. 4.

We define the nominal plasma frequency of the Yukawa system as $\omega_{p}=\sqrt{Q^{2} n / \epsilon_{0} m}$, where $m$ is the mass of a Yukawa particle. This represents the typical frequency of collective particle oscillation only if the interparticle potential is of (unscreened) Coulomb (i.e., $\kappa=0$ ). In the case of finite screening (i.e., $\kappa>0$ ), $\omega_{p}$ does not bear any particular physical significance. We also define the Einstein frequency by

$$
\omega_{\mathrm{E}}^{2}=\frac{1}{3 m} \sum_{i \neq j} \Delta \phi\left(\mathbf{r}_{i}-\mathbf{r}_{j}\right)=\frac{k_{D}^{2}}{3 m} \sum_{i \neq j} \phi\left(\mathbf{r}_{i}-\mathbf{r}_{j}\right),
$$


TABLE I. The critical $\Gamma$ for the fluid-solid phase transition and the fcc Einstein frequencies. From Refs. 3 and 4.

\begin{tabular}{ccc}
\hline \hline$\kappa$ & $\Gamma_{m}$ & $\sqrt{3} \omega_{\mathrm{E}} / \omega_{p}$ \\
\hline 0.0 & 171.8 & 1.0000 \\
0.1 & 172.2 & 0.9972 \\
0.3 & 175.7 & 0.9771 \\
0.6 & 187.1 & 0.9209 \\
1.0 & 217.4 & 0.8178 \\
1.4 & 268.8 & 0.7018 \\
2.0 & 440.1 & 0.5315 \\
3.0 & 1185 & 0.3047 \\
5.0 & $1.506 \times 10^{4}$ & 0.0810 \\
\hline \hline
\end{tabular}

where $\phi$ is the Yukawa potential of Eq. (1), the sum is taken over all $i$ except for (fixed) $j$ and all particles are assumed to be at given crystal structure sites. This represents the harmonic oscillation frequency of a particle around its equilibrium site when all other particles are located at their equilibrium sites. Note that $\omega_{\mathrm{E}} \rightarrow \omega_{p} / \sqrt{3}$ as $\kappa \rightarrow 0 .{ }^{19}$ Although $\omega_{\mathrm{E}}$ depends on the selected crystal structure, its numerical values for the fcc and bcc crystals differ by only less than $1 \%$. Therefore, in what follows, we shall use only the fcc Einstein frequencies for convenience. Table I lists the fcc Einstein frequencies for selected $\kappa$ values.

Particles in Yukawa systems in thermodynamical equilibrium travel under the influence of collisions with other particles. Such motions are called self-diffusion as the only forces exerted on each particle are those from other particles of the same kind. For charged dust particles in a plasma or colloidal particles in an electrolyte, their actual diffusion is not determined only by self-diffusion: motions of those particles are also affected by collisions with smaller particles comprising the background media (e.g., neutral atoms and molecules of the background gas in the first case). Furthermore, deviation of the interparticle potential from the Yukawa form due to, e.g., the wake field potential ${ }^{11,12}$ in a plasma mentioned previously, can significantly change the values of self-diffusion coefficients obtained in this paper. However, together with other transport coefficients such as viscosity and thermal conductivity, the self-diffusion coefficient is one of the most fundamental dynamical parameters that reflect the nature of interparticle potentials and characterize thermodynamics of the system. Therefore, despite possible differences between the self-diffusion coefficients and actual diffusion coefficients in those physical systems, we still think it is worthwhile to determine numerical values of the self-diffusion coefficient in the simplest possible model. In this paper, we evaluate the self-diffusion coefficients of Yukawa systems in the fluid phase, using molecular dynamics (MD) simulation. Prior to our study, Yukawa selfdiffusion coefficients were evaluated in a limited parameter range by several other authors. ${ }^{7-9,20-22}$ Our goal is therefore to present numerical values of the self-diffusion coefficients in a more systematic manner in a wider range of the system parameters.

\section{NUMERICAL SCHEMES}

Here we briefly discuss the numerical scheme of our MD simulation. Let us consider a system of $N$ identical particles with mass $m$ interacting through Eq. (1). To emulate the infinitely large system, the simulation particles are placed in a cubic box of side $L$ and periodic boundary conditions are imposed on all boundaries. Each particle then interacts with all the other particles in the simulation box and all of their periodic images. The effect of such image particles are important especially if the screening length $k_{D}^{-1}$ is comparable with or greater than the box size $L$. Then the effective pair potential $^{5}$ for actual simulation particles may be given by

$$
\Phi(\mathbf{r})=\phi(|\mathbf{r}|)+\sum_{\mathbf{n} \neq \mathbf{0}} \phi(|\mathbf{r}+\mathbf{n} L|),
$$

with $\phi(r)$ being the Yukawa potential, i.e., Eq. (1). The above-mentioned potential above represents the interaction energy of particle $i$ with particle $j$ (at separation $\mathbf{r}=\mathbf{r}_{j}-\mathbf{r}_{i}$ ) and with all periodic images of the latter. The infinite sum of $\phi$ over integer vectors $\mathbf{n}=(l, m, n)$ represents the contribution from all periodic images. In our MD simulation, the infinite sum of Eq. (2) is approximated numerically by a tensor-product spline function. ${ }^{23}$

To have the system attain the desired temperature $T$ (or $\Gamma$ ), we periodically rescale the velocity of each particle during the simulation until the system reaches the thermodynamical equilibrium. ${ }^{3-5}$ Once the system reaches thermodynamical equilibrium, we discontinue the periodic renormalization of particle velocities and let the system evolve under the constant-energy conditions (i.e., microcanonical simulation). In such a microcanonical MD simulation, the system temperature $T$ fluctuates but its mean value remains almost constant. The statistical average \langle\rangle of dynamical quantities is then obtained by taking the time average over a sufficiently long time period in the constant-energy simulation (i.e., microcanonical ensemble). The MD code used in this work was originally developed by R. T. Farouki ${ }^{5}$ and modified by the authors to calculate various time correlation functions.

\section{VELOCITY AUTOCORRELATION FUNCTION}

In this section we discuss the velocity autocorrelation function (VAF), which we use to evaluate self-diffusion coefficients. The VAF $Z(t)$ is defined as

$$
Z(t)=\left\langle\mathbf{v}_{j}(t) \cdot \mathbf{v}_{j}(0)\right\rangle,
$$

where $\mathbf{v}_{j}(t)$ is the velocity of the $j$ th particle at time $t$. As $Z(t)$ should be independent of the choice of a specific particle in thermodynamical equilibrium, we take the average over all particles in order to minimize statistical errors: ${ }^{24}$

$$
\begin{aligned}
Z(t) & =\frac{1}{N}\left\langle\sum_{i=1}^{N} \mathbf{v}_{i}(s) \cdot \mathbf{v}_{i}(s+t)\right\rangle \\
& =\frac{1}{M N} \sum_{k=1}^{M} \sum_{i=1}^{N}\left[\mathbf{v}_{i}\left(t_{k}\right) \cdot \mathbf{v}_{i}\left(t_{k}+t\right)\right] .
\end{aligned}
$$



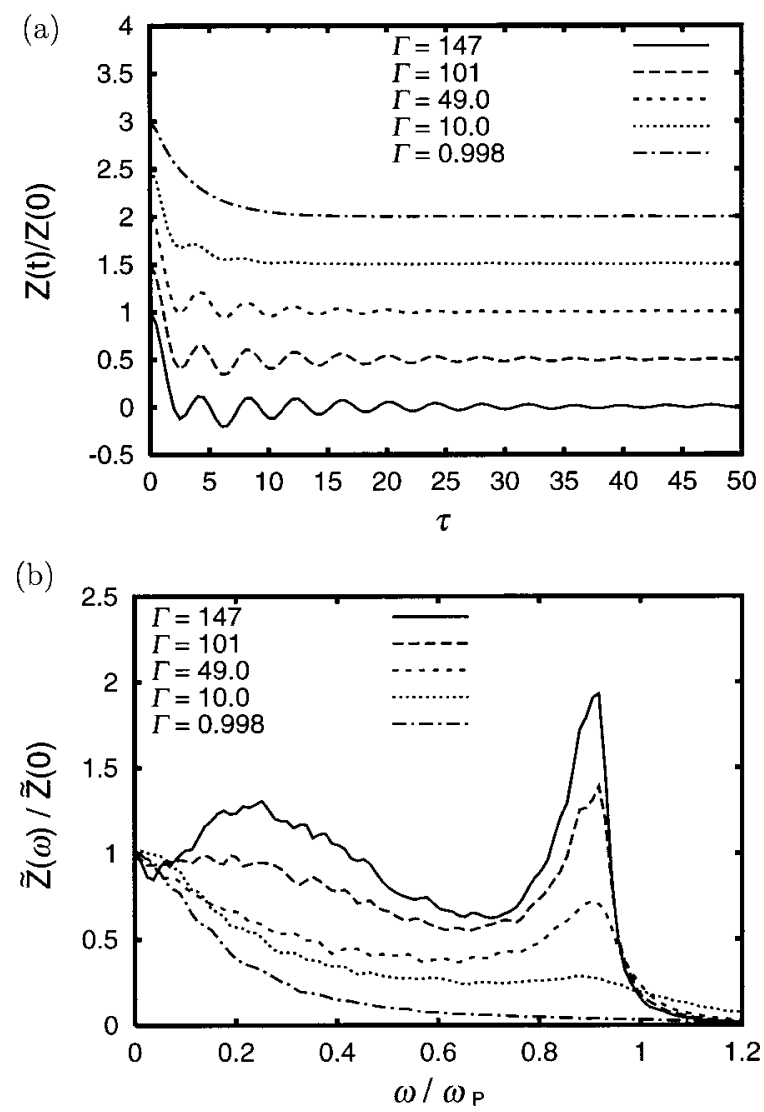

FIG. 1. (a) Velocity autocorrelation function $Z(t)$ and (b) its power spectrum $\widetilde{Z}(\omega)$ at $\kappa=0.3$ for various $\Gamma$ values. Note that curves in (a) are displaced vertically for clarity and $Z(t) \rightarrow 0$ for all $\Gamma$.

Here the ensemble average \langle\rangle is replaced by time average and $\left\{t_{1}, t_{2}, \ldots, t_{M}\right\}$ with $t_{k}=k \Delta$ denoting an equally spaced time sequence with the sampling period $\Delta$. In our simulation, we typically use $\Delta=\sqrt{3} / 2 \omega_{p}^{-1}$ and $M=800$. We optimize our simulation by varying the number of simulation particles $N$ from 250 to 1000 to achieve the best compromise between good accuracy and high computational efficiency.

We have also evaluated its Fourier transform $\widetilde{Z}(\omega)$ by directly integrating $Z(t)$ via

$$
\widetilde{Z}(\omega)=\frac{1}{2 \pi} \int_{-\infty}^{\infty} \exp (i \omega t) Z(t) d t .
$$

Figures 1 and 2 show $Z(t)$ and $\widetilde{Z}(\omega)$ of Yukawa systems in the fluid phase for $\kappa=0.3$ and 3.0 obtained from MD simulations with $N=300$ simulation particles. Note that, in Figs. 1(a) and 2(a), curves are displaced vertically for clarity and $Z(t) \rightarrow 0$ for all $\kappa$ and $\Gamma$.

It is shown in these figures that the VAFs are monotonically decreasing in time if $\Gamma$ is sufficiently small (e.g., $\Gamma$ $\leq \Gamma_{m} / 100$ ). In this regime, short time correlations are weak due to the high temperatures. The power spectrum for larger $\Gamma$ (however, $\Gamma<\Gamma_{m}$, i.e., the system is in the fluid phase) has two peaks. The peaks are prominent if the system is close to the fluid-solid phase transition, i.e., $\Gamma \simeq \Gamma_{m}$. As in the case of OCPs ${ }^{25}$ the peak at the higher frequency is related to the longitudinal wave (ion-acoustic mode or dust-acoustic wave if the Yukawa particles are viewed as ions or dust
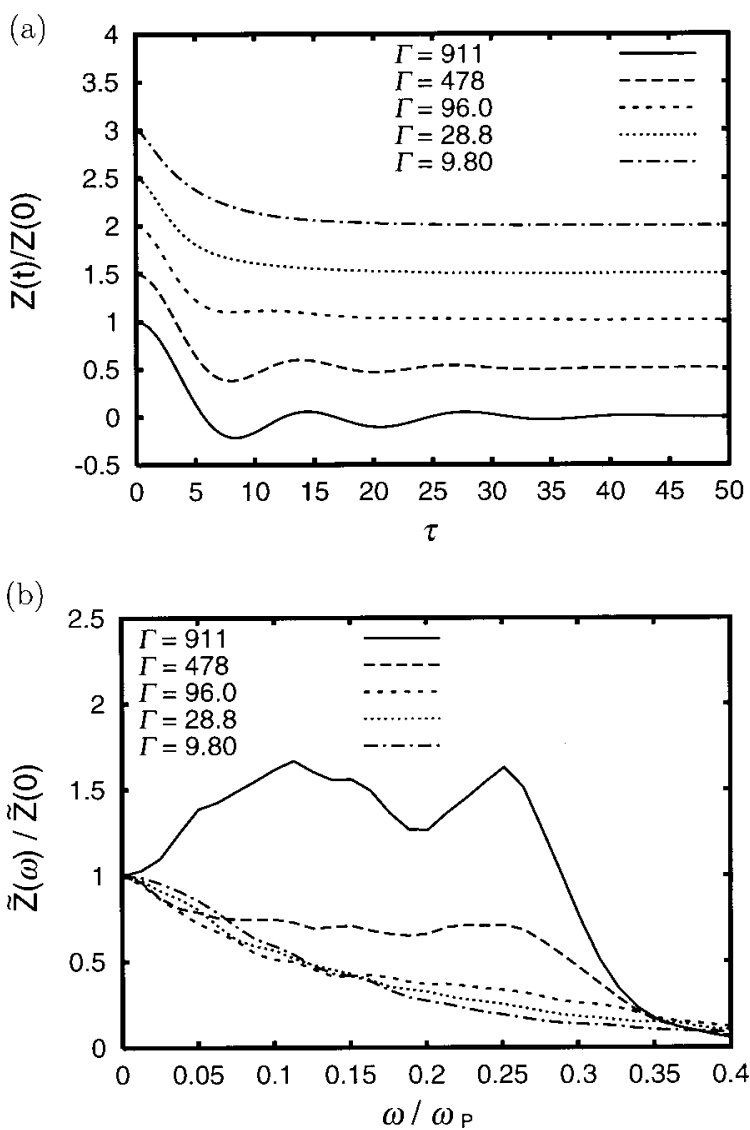

FIG. 2. (a) Velocity autocorrelation function $Z(t)$ and (b) its power spectrum $\widetilde{Z}(\omega)$ at $\kappa=3.0$ for various $\Gamma$ values. Note that curves in (a) are displaced vertically for clarity and $Z(t) \rightarrow 0$ for all $\Gamma$.

particles) whereas the broad peak at the lower frequency is related to the transverse wave (shear wave), the details of which will be discussed in Sec. V.

\section{SELF-DIFFUSION COEFFICIENTS}

The self-diffusion coefficient $D$ of a particle system may be evaluated from the Einstein relation

$$
D=\lim _{t \rightarrow 0} \frac{1}{6 t}\left\langle\left|\mathbf{r}_{j}(t)-\mathbf{r}_{j}(0)\right|^{2}\right\rangle,
$$

where $\mathbf{r}_{j}(t)$ represents the position of the $j$ th particle. As in Eq. (3), the above-mentioned statistical average is evaluated numerically as

$$
\left\langle\left|\mathbf{r}_{j}(t)-\mathbf{r}_{j}(0)\right|^{2}\right\rangle=\frac{1}{M N} \sum_{k=1}^{M} \sum_{i=1}^{N}\left[\left|\mathbf{r}_{i}\left(t_{k}+t\right)-\mathbf{r}_{i}\left(t_{k}\right)\right|^{2}\right]
$$

for the same discrete time sequence $\left\{t_{1}, t_{2}, \ldots t_{M}\right\}$. It is easy to show $^{26}$ that the self-diffusion coefficient is related to the velocity autocorrelation function $Z(t)$ as

$$
D=\frac{1}{3} \int_{0}^{\infty} Z(t) d t,
$$

which is known as the Green-Kubo formula.

One can use either Eq. (4) or Eq. (5) to evaluate $D$ from MD simulations. We have calculated $D_{E}(t) \equiv\langle| \mathbf{r}_{j}(t)$ 
TABLE II. Self-diffusion coefficients obtained from MD simulations with $N=300$. The normalized diffusion coefficients are defined by $D_{E}^{*}=D_{E} / \omega_{\mathrm{E}} a^{2}$ and $D_{Z}^{*}=D_{Z} / \omega_{\mathrm{E}} a^{2}$.

\begin{tabular}{|c|c|c|c|c|c|c|c|c|c|}
\hline$\kappa$ & $\Gamma$ & $T^{*}$ & $D_{E}^{*}$ & $D_{Z}^{*}$ & $\kappa$ & $\Gamma$ & $T^{*}$ & $D_{E}^{*}$ & $D_{Z}^{*}$ \\
\hline \multirow[t]{11}{*}{0.1} & 150 & 1.14 & 0.00486 & 0.00477 & & 4.76 & 45.7 & 0.654 & 0.621 \\
\hline & 98.9 & 1.74 & 0.0112 & 0.0111 & & 2.92 & 74.4 & 1.25 & 1.27 \\
\hline & 50.0 & 3.44 & 0.0351 & 0.0357 & & 1.96 & 111 & 1.98 & 2.08 \\
\hline & 30.2 & 5.70 & 0.0627 & 0.0626 & & & & & \\
\hline & 10.4 & 16.5 & 0.206 & 0.205 & 1.4 & 232 & 1.16 & 0.00593 & 0.00591 \\
\hline & 6.86 & 25.1 & 0.317 & 0.314 & & 147 & 1.83 & 0.0145 & 0.0141 \\
\hline & 5.03 & 34.2 & 0.427 & 0.439 & & 97.8 & 2.75 & 0.0274 & 0.0281 \\
\hline & 3.06 & 56.3 & 0.775 & 0.786 & & 47.7 & 5.63 & 0.0698 & 0.0693 \\
\hline & 2.00 & 86.3 & 1.38 & 1.38 & & 18.9 & 14.2 & 0.202 & 0.207 \\
\hline & 0.987 & 174 & 3.35 & 3.34 & & 9.35 & 28.7 & 0.412 & 0.410 \\
\hline & & & & & & 4.80 & 56.0 & 0.815 & 0.834 \\
\hline \multirow[t]{10}{*}{0.3} & 147 & 1.19 & 0.00617 & 0.00620 & & 2.94 & 91.5 & 1.62 & 1.60 \\
\hline & 101 & 1.75 & 0.0113 & 0.0111 & & 2.01 & 134 & 2.64 & 2.66 \\
\hline & 49.0 & 3.59 & 0.0376 & 0.0381 & & & & & \\
\hline & 28.8 & 6.10 & 0.0713 & 0.0731 & 2.0 & 374 & 1.18 & 0.00604 & 0.00598 \\
\hline & 10.0 & 17.5 & 0.223 & 0.229 & & 185 & 2.38 & 0.0220 & 0.0215 \\
\hline & 5.01 & 35.1 & 0.455 & 0.461 & & 96.0 & 4.59 & 0.0566 & 0.0555 \\
\hline & 3.00 & 58.6 & 0.805 & 0.831 & & 46.3 & 9.51 & 0.130 & 0.134 \\
\hline & 2.00 & 87.8 & 1.35 & 1.33 & & 18.8 & 23.4 & 0.310 & 0.318 \\
\hline & 0.998 & 176 & 3.65 & 3.63 & & 9.42 & 46.7 & 0.657 & 0.660 \\
\hline & & & & & & 4.94 & 89.0 & 1.48 & 1.49 \\
\hline \multirow[t]{10}{*}{0.6} & 142 & 1.32 & 0.00654 & 0.00623 & & & & & \\
\hline & 119 & 1.58 & 0.00973 & 0.0101 & 3.0 & 911 & 1.30 & 0.00768 & 0.00742 \\
\hline & 67.8 & 2.76 & 0.0241 & 0.0243 & & 478 & 2.48 & 0.0256 & 0.0246 \\
\hline & 28.5 & 6.57 & 0.0851 & 0.0825 & & 96.0 & 12.3 & 0.175 & 0.175 \\
\hline & 19.5 & 9.59 & 0.126 & 0.127 & & 47.1 & 25.2 & 0.331 & 0.348 \\
\hline & 9.86 & 19.0 & 0.244 & 0.244 & & 28.8 & 41.1 & 0.545 & 0.568 \\
\hline & 3.81 & 49.1 & 0.698 & 0.701 & & 19.0 & 62.5 & 0.814 & 0.827 \\
\hline & 2.91 & 64.2 & 0.958 & 1.02 & & 9.80 & 121 & 1.71 & 1.67 \\
\hline & 1.98 & 94.6 & 1.64 & 1.69 & & & & & \\
\hline & & & & & 5.0 & 12300 & 1.22 & 0.00666 & 0.00684 \\
\hline \multirow[t]{8}{*}{1.0} & 195 & 1.12 & 0.00510 & 0.00467 & & 6420 & 2.35 & 0.0178 & 0.0185 \\
\hline & 143 & 1.52 & 0.00946 & 0.00982 & & 960 & 15.7 & 0.155 & 0.153 \\
\hline & 65.0 & 3.35 & 0.0334 & 0.0340 & & 484 & 31.1 & 0.320 & 0.328 \\
\hline & 38.8 & 5.61 & 0.0692 & 0.0721 & & 286 & 52.6 & 0.510 & 0.533 \\
\hline & 28.2 & 7.71 & 0.0953 & 0.0911 & & 241 & 62.4 & 0.573 & 0.611 \\
\hline & 18.9 & 11.5 & 0.152 & 0.154 & & 170 & 88.8 & 0.795 & 0.817 \\
\hline & 9.55 & 22.8 & 0.311 & 0.325 & & 142 & 106 & 0.940 & 0.971 \\
\hline & & & & & & 137 & 110 & 0.969 & 1.03 \\
\hline
\end{tabular}

$\left.-\left.\mathbf{r}_{j}(0)\right|^{2}\right\rangle / 6 t$ and $D_{Z}(t) \equiv \int_{0}^{t} Z(t) d t / 3$ as functions of time $t$ and observed that $D_{E}(t)$ and $D_{Z}(t)$ converged to a single value in most cases if $\tau \equiv \omega_{p} t / \sqrt{3}>100$. Therefore, to evaluate $D$, we took the time average of $D_{E}(t)$ and $D_{Z}(t)$ typically over $100<\tau<500$. The self-diffusion coefficients obtained in this manner are listed in Table II [where $D_{E}^{*}$ $=D_{E} / \omega_{\mathrm{E}} a^{2}$ and $D_{Z}^{*}=D_{Z} / \omega_{\mathrm{E}} a^{2}$ with $D_{E}=\lim _{t \rightarrow \infty} D_{E}(t)$ and $D_{Z}=\lim _{t \rightarrow \infty} D_{Z}(t)$. Theoretically $D \equiv D_{E}=D_{Z}$, as mentioned previously.] Here the diffusion coefficients are normalized by $\omega_{\mathrm{E}} a^{2}$ with $\omega_{\mathrm{E}}$ being the Einstein frequency for the fcc crystals. ${ }^{9}$ As in Sec. III, we have employed $N=300$ particles for these MD simulations.

Denoting $D^{*}=D / \omega_{\mathrm{E}} a^{2}$, we fit the data given in Table II to the form

$$
D^{*}=\alpha\left(T^{*}-1\right)^{\beta}+\gamma,
$$

for each $\kappa$. Here $T^{*}$ is the ratio of the system temperature $T$ to the fluid-solid critical temperature $T_{m}$ (i.e., melting temperature), i.e., $T^{*} \equiv T / T_{m}=\Gamma_{m} / \Gamma$ with $\Gamma_{m}=Q^{2} / 4 \pi \epsilon_{0} a T_{m}$. As the system under consideration is in the fluid phase, we have $T^{*}>1$. For each $\kappa$, the least-squares fitting parameters $\alpha, \beta$, and $\gamma$ are given in Table III. The fitting parameters for $\kappa=0$ in Table III were obtained from least-squares fitting Eq. (6) to the OCP simulation data by Hansen et al. ${ }^{14}$ As shown in Fig. 3 for some selected $\kappa$, Eq. (6) is an excellent fitting formula for the simulation data. As $\kappa$ varies from 0 to 5, the values of $\omega_{\mathrm{E}}$ and $\Gamma_{m}$ vary by more than the order of magni-

TABLE III. The fitting parameters for the self-diffusion coefficients given by Eq. (6).

\begin{tabular}{llcc}
\hline \hline$\kappa$ & \multicolumn{1}{c}{$\alpha$} & $\beta$ & $\gamma$ \\
\hline 0 & 0.00913 & 1.15 & 0.00457 \\
0.1 & 0.0104 & 1.09 & 0.00364 \\
0.3 & 0.0106 & 1.09 & 0.00429 \\
0.6 & 0.0122 & 1.06 & 0.00282 \\
1.0 & 0.0121 & 1.07 & 0.00367 \\
1.4 & 0.0125 & 1.07 & 0.00419 \\
2.0 & 0.0131 & 1.04 & 0.00385 \\
3.0 & 0.0156 & 0.97 & 0.00265 \\
5.0 & 0.0112 & 0.96 & 0.00399 \\
\hline \hline
\end{tabular}


(a) $\kappa=0.1$

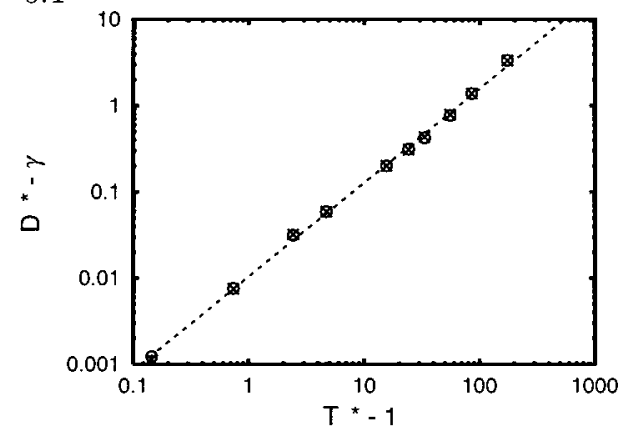

(b) $\kappa=1.4$

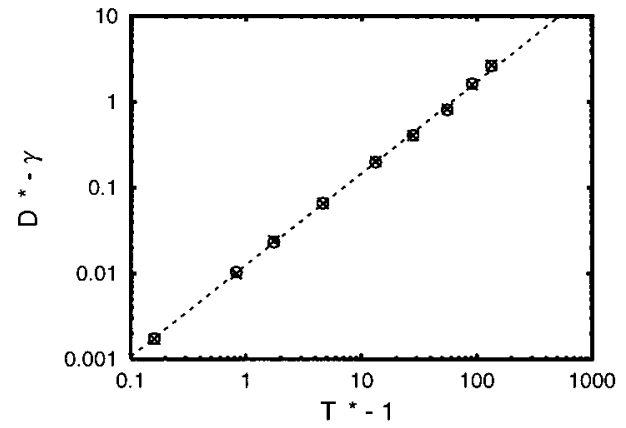

(c) $\kappa=5.0$

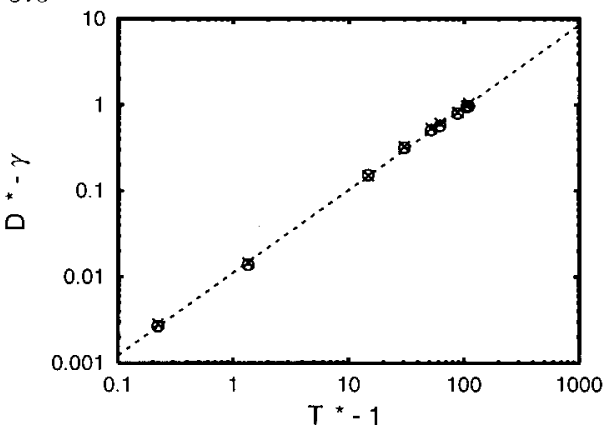

FIG. 3. Fitting of Eq. (6) to the self-diffusion coefficients: (a) $\kappa=0.1$ and $\gamma=0.00364$, (b) $\kappa=1.4$ and $\gamma=0.00419$, (c) $\kappa=5.0$ and $\gamma=0.003$ 99. The symbols $\bigcirc$ and $\times$ represent $D_{E}^{*}$ and $D_{Z}^{*}$ given in Table II and other fitting parameters are given in Table III.

tude. Compared with this variation, the variation of fitting parameters $\alpha, \beta$, and $\gamma$ over the same range of $\kappa$ is relatively small.

Figures 4 and 5 plot values of $D^{*}$ given in Table II for various $\kappa$. [Figure 4(a) is an expansion of the lower lefthand corner of Fig. 4(b).] Robbins, Kremer, and Grest found that, ${ }^{9}$ if the system is relatively close to melting, values of $D$ for all $\kappa$ can be given by a single universal scaling law. The data by Robbins et al..$^{9}$ are in a limited parameter range $\left(2.0 \leqslant \kappa \leqslant 6.3,0.5 \leqslant T^{*} \leqslant 2\right.$, where $0.5 \leqslant T^{*}<1$ is for supercooled states), but as can be seen in Fig. 4, this universal scaling may be extended to $T^{*} \simeq 10$ in the fluid phase. The dashed line represents the least-squares fit of a linear function (i.e., $\beta=1$ ) of $T^{*}$ to the data of Fig. 4(b), i.e., Eq. (6) with $\alpha=0.0132, \beta=1$, and $\gamma=0.00317$. This scaling is consistent with simulation results by Robbins et al. ${ }^{9}$ This scaling also seems to be consistent with the universal entropy scaling of the self-diffusion coefficients. ${ }^{27,28}$ However detailed dis-
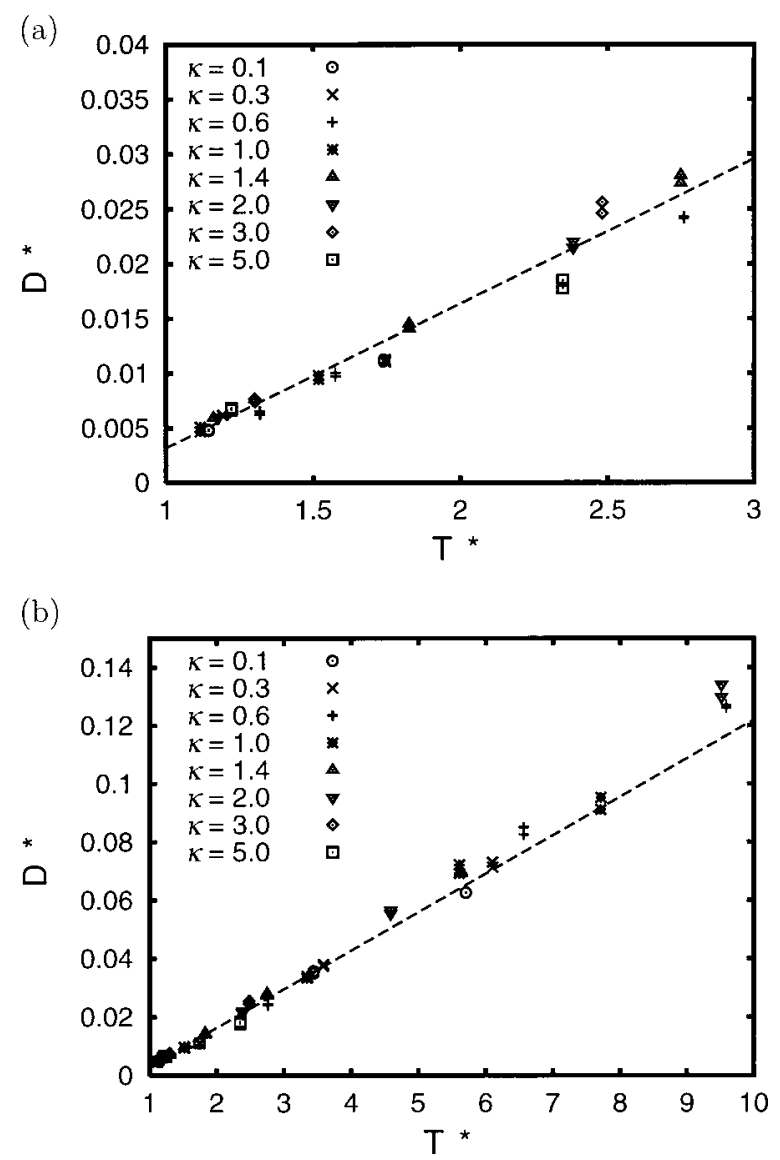

FIG. 4. Self-diffusion coefficient $D\left(D_{E}^{*}\right.$ and $D_{Z}^{*}$, as given in Table II) vs normalized temperature $T^{*}$ for (a) $1 \leqslant T^{*} \leqslant 3$ and (b) $1 \leqslant T^{*} \leqslant 10$. The dashed lines in both (a) and (b) are the linear least-squares fit to the data (listed in Table II) for $1 \leqslant T^{*} \leqslant 10$.

cussion on the entropy scaling will be presented in future publications.

The fact that $D^{*}$ is independent of $\kappa$ for $1<T^{*} \leq 10$ may be accounted for in the following manner. When a fluid system is close to solidification, the motion of each particle may be regarded as oscillation about its equilibrium site and par-



FIG. 5. Self-diffusion coefficient $D$ for $\kappa=0.1$ and $5.0\left(D_{E}^{*}\right.$ and $D_{Z}^{*}$, as given in Table II) vs normalized temperature $T^{*}$. The dashed and dotdashed curves represent the fitting curves given by Eq. (6) with the corresponding fitting parameters given in Table III. 
ticle diffusion results from occasional hopping motion of the particle from one equilibrium site to another. Such a selfdiffusion process may be characterized by the diffusion coefficient given by $D=C \Delta r^{2} / \Delta t$, where $C$ is a proportional constant, $\Delta r$ is the oscillation amplitude, and $\Delta t=\omega_{\mathrm{E}}^{-1}$ is the typical time scale of oscillation. The Lindemann criterion ${ }^{29}$ states that fluid-solid phase transition occurs when the ratio $\Delta r / a$ reaches a universal constant regardless of the form of interparticle potentials. Therefore, if the fluid system is near the phase transition (i.e., $T^{*}$ is close to 1 ), the systems of the same $T^{*}$ are likely to have the same ratio $R=\Delta r / a$, regardless of $\kappa$. Under this ansatz, we may write $D^{*} \propto D / \omega_{\mathrm{E}} a^{2}$ $=C R^{2}$, which is independent of $\kappa$ for a given $T^{*}$.

For higher temperatures, the correlation among particles becomes weak and the particle diffusion is governed more by two-body collisions. As noted by Hansen et al. ${ }^{14}$ for OCP and by Rosenberg et al. ${ }^{7}$ for a Yukawa system, the relation between $D^{*}$ and $T$ (or $T^{*}$ ) is no longer linear for larger $T$. In Table III we observe the tendency that $\beta$ decreases as $\kappa$ increases. This slight dependence of $\beta$ on $\kappa$ manifests itself in the dependence of $D^{*}$ on $T^{*}$ for large $T^{*}$, as shown in Fig. 5.

We now briefly comment on the accuracy of the selfdiffusion coefficients presented in this work. As one can see in Figs. 3-5, the obtained data are somewhat scattered around the fitting curves, which suggests that the numerical values of $D$ given in Table II may have errors of up to about $10 \%$. The possible sources of these uncertainties include; (a) the simulation system may not be completely in thermal equilibrium, (b) $D_{E}(t)$ given by Eq. (4) may not have completely converged yet, (c) $D_{Z}(t)$ may contain errors arising from the numerical evaluation of the integral (5) for large $t$, where the integrand $Z(t)$ is nearly zero, and (d) if $\Gamma$ is extremely large (e.g., $\gtrsim 10^{4}$ ), then the system (even in thermal equilibrium) suffers noticeable temperature shift during the microcanonical simulation due to discretization errors in time integration. To minimize effects of such temperature shift, we take the time average of system temperatures and also use the time average of $D_{E}(t)$, rather than the actual limit $\lim _{t \rightarrow \infty} D_{E}(t)$, as mentioned before.

On the other hand, a possible source of systematic errors in the numerically evaluated self-diffusion coefficients is the $N$ dependence. As is known for particle systems with other potentials, the numerical self-diffusion coefficient values depend on the number of simulation particle $N .{ }^{30}$ However, in our case, the correction of $D$ due to the $N$ dependence seems comparable with errors due to (a) - (d) mentioned previously. For example, from MD simulation for $\kappa=0.1$, we have obtained $D_{E} / \omega_{\mathrm{E}} a^{2}=0.00486 \quad$ (at $\Gamma=150$ ), $0.00562 \quad$ (at $\Gamma=148$ ), and 0.00534 (at $\Gamma=150$ ) for $N=300,600$, and 1000 , respectively. Similarly, $D_{Z} / \omega_{\mathrm{E}} a^{2}=0.00477$ (at $\Gamma=150$ ), 0.00560 (at $\Gamma=148$ ), and 0.00537 (at $\Gamma=150$ ) for $N=300,600$, and 1000 , respectively. Other possible systematic errors due to, for example, the shape of the boundaries are not examined here. ${ }^{31}$

\section{MODE COUPLING THEORY}

Schmidt et al. $^{25}$ have shown that the power spectrum of the $\operatorname{VAF} \widetilde{Z}(\omega)$ for a fluid OCP exhibits two peaks related to the excitation of longitudinal and transverse waves if the system is close to solidification. In this section, we shall show that the same holds for Yukawa systems, using the mode-coupling theory ${ }^{25,26}$ For a given wave number $\mathbf{k}$, let us define the longitudinal and transverse current correlation functions ${ }^{26}$ as

$$
C_{l}(\mathbf{k}, t)=\frac{1}{N}\langle[\mathbf{k} \cdot \mathbf{j}(\mathbf{k}, t)][\mathbf{k} \cdot \mathbf{j}(-\mathbf{k}, 0)]\rangle
$$

and

$$
C_{t}(\mathbf{k}, t)=\frac{1}{2 N}\langle[\mathbf{k} \times \mathbf{j}(\mathbf{k}, t)] \cdot[\mathbf{k} \times \mathbf{j}(-\mathbf{k}, 0)]\rangle .
$$

Here

$$
\mathbf{j}(\mathbf{k}, t)=\sum_{j=1}^{N} \mathbf{v}_{i}(t) \exp \left[i \mathbf{k} \cdot \mathbf{r}_{j}(t)\right]
$$

is the Fourier transformation of the microscopic particle current

$$
\mathbf{j}(\mathbf{r}, t)=\sum_{j=1}^{N} \mathbf{v}_{j}(t) \delta\left[\mathbf{r}-\mathbf{r}_{j}(t)\right] .
$$

We also write the Fourier transformation of these functions into the frequency space as $\widetilde{C}_{l}(\mathbf{k}, \omega)$ and $\widetilde{C}_{t}(\mathbf{k}, \omega)$. Waves excited in Yukawa systems are collective motions of the constituent particles and can be characterized by these correlation functions.

We have used MD simulations with $N=250$ simulation particles to evaluate the current correlation functions. As the simulation volume is finite, the wave numbers $\mathbf{k}$ that can be examined in our simulations are limited to

$$
\mathbf{q}=\left(\frac{2 \pi}{L / n_{1}}, \frac{2 \pi}{L / n_{1}}, \frac{2 \pi}{L / n_{1}}\right)
$$

with $\left(n_{1}, n_{2}, n_{3}\right)$ being the integer triplet. Since the system is isotropic, the correlation functions depend only on the magnitude of the wave number, i.e., $k=|\mathbf{k}|$. Therefore the smallest wave number $k_{\min }$ that we can take in our MD simulations is given by $k_{\min }=2 \pi / L$. (For $N=250$ particles, we have $k_{\min } a \simeq 0.619$.)

We have obtained the power spectra $\widetilde{C}_{l}(\mathbf{k}, \omega)$ and $\widetilde{C}_{t}(\mathbf{k}, \omega)$ of the current correlation functions from the fast Fourier transform (FFT) of the MD simulation data. Generally the straightforward application of the FFT to MD data results in low signal/noise ratios and therefore some smoothing of FFT spectra is required. To evaluate the power spectra of the current correlation functions, we first equally divided 1920 discrete time-sequential data into 15 sets, applied FFT to each data set, and then took the average over the obtained 15 FFT spectra. This process limits the frequency resolution to $\Delta \omega=0.0283 \omega_{p}$ in power spectrums obtained from the FFT in this paper. To further reduce statistical noise, we also averaged the correlation functions over different wave vectors of the same magnitude $k=|\mathbf{k}|$. For example, for a given wave number vector $\mathbf{k}=\left(k_{1}, k_{2}, k_{3}\right)$, all of its permutations such as $\left(k_{1}, k_{3}, k_{2}\right),\left(k_{2}, k_{1}, k_{3}\right), \cdots$, are considered to be equivalent for the current correlation functions since the sys- 


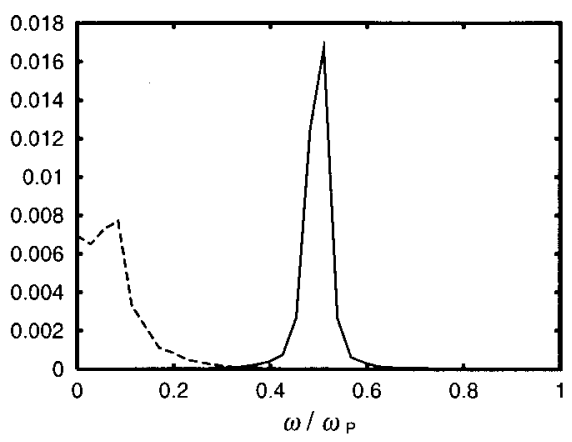

(a) $q=0.619$

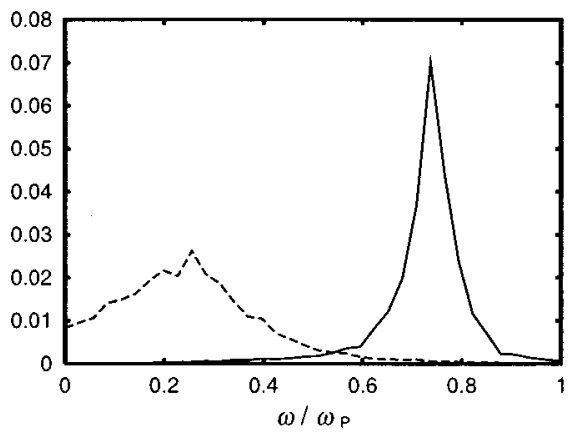

(b) $q=1.24$

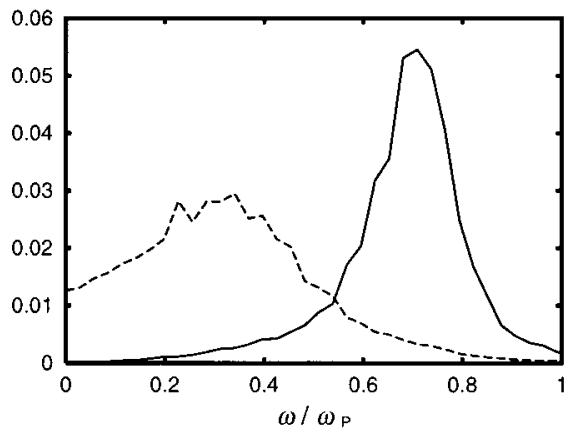

(c) $q=1.75$

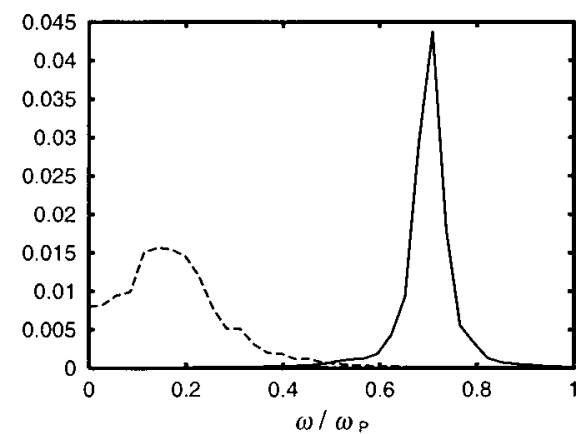

(d) $q=1.96$



(e) $q=2.23$



(f) $q=2.48$
FIG. 6. Power spectra of longitudinal and transverse current correlation functions, i.e., $\widetilde{C}_{l}(k, \omega)$ (denoted by solid curves) and $\widetilde{C}_{t}(k, \omega)$ (denoted by dotted curves) for various wave numbers $k(q=k a)$ for $\kappa=1.0$ and $\Gamma=202$ [coupling parameter $\Gamma^{*}=\Gamma \exp (-\kappa)$ $=74.3]$. tem is isotropic. In general, the power spectrum of the autocorrelation function $C_{A A}(t) \equiv\langle A(t) A(0)\rangle$ of a function $A(t)$ may be given by

$$
\widetilde{C}_{A A}(\omega)=\lim _{T \rightarrow \infty} \frac{2 \pi}{T} \widetilde{A}_{T}(\omega) \widetilde{A}_{T}^{*}(\omega)=\lim _{T \rightarrow \infty} \frac{2 \pi}{T}\left|\widetilde{A}_{T}(\omega)\right|^{2},
$$

where

$$
\widetilde{A}_{T}(\omega)=\frac{1}{\omega} \int_{-T / 2}^{T / 2} \exp (i \omega t) A(t) d t
$$

Therefore, to obtain the power spectra of the current autocorrelations we first obtain $\widetilde{A}_{T}(\omega)\left[\right.$ e.g., $\widetilde{A}_{T}(\omega)=\widetilde{\mathbf{j}}(\mathbf{k}, \omega) \cdot \mathbf{k}$ for $\left.\widetilde{C_{l}}(\mathbf{k}, \omega)\right]$ using FFT for a sufficiently large $T$. To minimize nonphysical effects arising from the finiteness of $T$, we apply a smooth data window edged with cosine functions to the original time-sequential discrete data before applying FFT. The magnitude of the thus obtained FFT power spectrum are then adjusted accordingly. ${ }^{32}$

Figure 6 shows the power spectra of longitudinal and transverse current correlation functions, i.e., $\widetilde{C}_{l}(k, \omega)$ (denoted by solid curves) and $\widetilde{C}_{t}(k, \omega)$ (denoted by dotted curves) for $\kappa=1.0$ and $\Gamma=202$ [coupling parameter $\Gamma^{*}$ $=\Gamma \exp (-\kappa)=74.3]$. The peaks of the current correlation functions give the linear dispersion relations for the corresponding waves. ${ }^{33,34}$

Similarly the self-intermediate scattering function is defined as

$$
S_{s}(\mathbf{k}, t)=\left\langle\rho_{s}(\mathbf{k}, t) \rho_{s}(-\mathbf{k}, 0)\right\rangle
$$

with

$$
\rho_{s}(\mathbf{k}, t)=\exp \left(i \mathbf{k} \cdot \mathbf{r}_{j}(t)\right)
$$




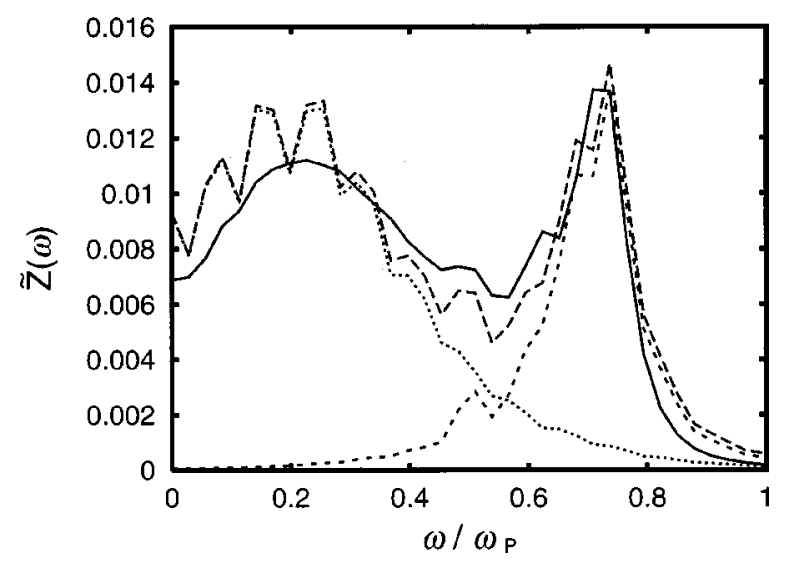

FIG. 7. The long-dashed curve is the VAF obtained from the mode-coupling theory, i.e., Eq. (9), for $\kappa=1.0$ and $\Gamma=202$. The contributions from the $\widetilde{C}_{l}(k, \omega)$ and $\widetilde{C}_{t}(k, \omega)$ are given by the short-dashed curve in the higher frequency side and the dotted curve in the lower frequency side. The solid curve is the power spectrum of the $\operatorname{VAF} \widetilde{Z}(\omega)$ directly obtained from $Z(t)$ via FFT. The jaggedness of the curves is due to statistical noise.

being the density of a single (the $j$ th) particle. As before, we denote the Fourier transform of $S_{s}(\mathbf{k}, t)$ in the frequency space by $\widetilde{S}_{s}(\mathbf{k}, \omega)$, which is called the self-dynamical structure factor. Then, from the mode-coupling theory, ${ }^{25,26}$ we have

$$
Z(t) \simeq \frac{1}{n(2 \pi)^{3}} \int \frac{d \mathbf{k}}{k^{2}} S_{s}(\mathbf{k}, t)\left[C_{l}(\mathbf{k}, t)+2 C_{t}(\mathbf{k}, t)\right]
$$

in the strongly coupled regime. Taking the Fourier transform of the above-mentioned equation, we obtain

$$
\begin{aligned}
\widetilde{Z}(\omega) \simeq & \frac{1}{n(2 \pi)^{3}} \int \frac{d \mathbf{k}}{k^{2}} \widetilde{S}_{s}(\mathbf{k}, \omega) *\left[\widetilde{C}_{l}(\mathbf{k}, \omega)\right. \\
& \left.+2 \widetilde{C}_{t}(\mathbf{k}, \omega)\right]
\end{aligned}
$$

where the asterisk denotes the convolution. Note the abovementioned integrals are divergent for large $k$. Since waves whose wavelengths are much shorter than the average interparticle distance are meaningless, we set the upper limit of the $k$ integration $^{25}$ as $k_{\max }=\left(6 \pi^{2} n\right)^{1 / 3}=2.42 / a$. The longdashed curve in Fig. 7 shows the power spectrum of the VAF constructed in this manner, i.e., the right-hand side of Eq. (9), for $\kappa=1.0$ and $\Gamma=202$. Here we have used the current correlation functions $\widetilde{C}_{l}(k, \omega)$ and $\widetilde{C}_{t}(k, \omega)$ shown in Fig. 6 and also $\widetilde{S}_{s}(k, \omega)$ obtained in a similar manner to evaluate Eq. (9). The contribution from the longitudinal current correlation function $\widetilde{C}_{l}(k, \omega)$ is given by the short-dashed curve in the higher frequency side whereas the contribution from the transverse current correlation function $\widetilde{C}_{t}(k, \omega)$ is given by the dotted curve in the lower frequency side. The sum of these curves is the upper dotted curve. The solid curve is the power spectrum of the $\operatorname{VAF} \widetilde{Z}(\omega)$ directly obtained from $Z(t)$ via FFT. The jaggedness of the curves are due to statistical noise. The agreement is good and this analysis shows that, as in the case of OCPs demonstrated by Schmidt et al. ${ }^{25}$ the peak in the higher frequency is accounted for by the excitation of the longitudinal mode whereas the broad peak in the lower frequency by that of the transverse mode (i.e., shear mode).

\section{CONCLUSIONS}

We have presented the self-diffusion coefficients of Yukawa fluids obtained from MD simulations in a wide range of the thermodynamical parameters $\kappa$ and $\Gamma$. The selfdiffusion coefficients are evaluated from both Einstein relation and Green-Kubo formula for the VAF. The numerically obtained self-diffusion coefficient $D$ is found to follow a simple scaling relation given by Eq. (6), where the dependence of coefficients $\alpha, \beta$, and $\gamma$ on $\kappa$ is relatively weak, as shown in Table III. Especially if the system temperature $T$ is close to the critical temperature $T_{m}$, the normalized self-diffusion coefficient $D^{*}\left(=D / \omega_{\mathrm{E}} a^{2}\right)$ is proportional to $T^{*}\left(=T / T_{m}\right)$, the coefficients of which are independent of $\kappa$. This universal linear scaling was previously observed by Robbins et al. ${ }^{9}$ in a relatively limited parameter range, but we have confirmed the linear scaling holds approximately in the range of $1<T / T_{m} \lesssim 10$ with good accuracy. We have also presented the VAFs and its power spectra as functions of the thermodynamical parameters. As in the case of OCPs demonstrated by Schmidt et al. ${ }^{25}$ it is shown that two peaks of the VAF's power spectrum in the strong coupling regime are associated with waves excited in the system.

In the case of dusty plasmas or colloidal suspensions, the diffusion of particulates is usually dominated by collisions with the background media (e.g., background neutral gas molecules/atoms in the case of dusty plasmas), rather than self-diffusion, as mentioned earlier. Therefore the selfdiffusion coefficients obtained in this work do not directly represent diffusivity observed in experiments of those systems. However, together with other transport coefficients such as viscosity and thermal conductivity, the self-diffusion coefficient is one of the most fundamental dynamical parameters that reflect the nature of the interparticle potentials and characterize thermodynamics of the system. Evaluation of other transport coefficients for Yukawa systems in the same parameter range is the subject of a future study.

\section{ACKNOWLEDGMENTS}

The authors thank R. T. Farouki for helpful discussion on MD numerical simulations. This work was in part supported by the Sumitomo Foundation.

${ }^{1}$ F. Hoyle and N. C. Wickramasinghe, The Theory of Cosmic Grains (Kluwer Academic, London, 1991).

${ }^{2}$ S. Hamaguchi, Plasmas \& Ions 2, 57 (1999).

${ }^{3}$ S. Hamaguchi, R. T. Farouki, and D. H. E. Dubin, Phys. Rev. E 56, 4671 (1997).

${ }^{4}$ S. Hamaguchi, R. T. Farouki, and D. H. E. Dubin, J. Chem. Phys. 105, 7641 (1996)

${ }^{5}$ R. T. Farouki and S. Hamaguchi, J. Chem. Phys. 101, 9885 (1994).

${ }^{6}$ S. Hamaguchi and R. T. Farouki, J. Chem. Phys. 101, 9876 (1994).

${ }^{7}$ R. O. Rosenberg and D. Thirumalai, Phys. Rev. A 33, 4473 (1986).

${ }^{8}$ K. Kremer, M. O. Robbins, and G. S. Grest, Phys. Rev. Lett. 57, 2694 (1986).

${ }^{9}$ M. O. Robbins, K. Kremer, and G. S. Grest, J. Chem. Phys. 88, 3286 (1988).

${ }^{10}$ U. Konopka, G. E. Morfill, and L. Ratke, Phys. Rev. Lett. 84, 891 (2000). 
${ }^{11}$ M. Nambu, S. V. Vladimirov, and P. K. Shukla, Phys. Lett. A 203, 40 (1995).

${ }^{12}$ S. V. Vladimirov and M. Nambu, Phys. Rev. E 52, 2172 (1995).

${ }^{13}$ S. G. Brush, H. L. Sahlin, and E. Teller, J. Chem. Phys. 45, 2102 (1966).

${ }^{14}$ J.-P. Hansen, I. R. McDonald, and E. L. Pollock, Phys. Rev. A 11, 1025 (1975).

${ }^{15}$ M. Baus and J.-P. Hansen, Phys. Rep. 59, 1 (1980).

${ }^{16}$ W. L. Slattery, G. D. Doolen, and H. E. DeWitt, Phys. Rev. A 21, 2087 (1980); 26, 2255 (1982).

${ }^{17}$ R. T. Farouki and S. Hamagauchi, Phys. Rev. E 47, 4330 (1993).

${ }^{18}$ The fitting function (26) of Ref. 4 together with the conversion formula (24) of Ref. 4 gives slightly different critical values. Note that there are typographical errors in Eq. (16) of Ref. 3 and Eq. (24) of Ref. 4, which should read $\mathcal{T}=(1 / \Gamma)(3 / 4 \pi)^{2 / 3}\left[(2 / 3) \kappa^{2} E_{\mathrm{fcc}}(\kappa)+\kappa^{3} / 3+1\right]^{-1}$.

${ }^{19}$ K. I. Golden, G. Kalman, and P. Wyns, Phys. Rev. A 46, 3454 (1992).

${ }^{20}$ G. Zerah, J. Clerouin, and E. L. Pollock, Phys. Rev. Lett. 69, 446 (1992).

${ }^{21}$ J. Clerouin, E. L. Pollock, and G. Zerah, Phys. Rev. A 46, 5130 (1992).

${ }^{22}$ Y. Rosenfeld, E. Nardi, and Z. Zinamon, Phys. Rev. Lett. 75, 2490 (1995).
${ }^{23}$ R. T. Farouki and S. Hamaguchi, J. Comput. Phys. 115, 276 (1994).

${ }^{24}$ J. M. Haile, Molecular Dynamics Simulation (Wiley-Interscience, New York, 1992).

${ }^{25}$ P. Schmidt, G. Zwicknagel, P.-G. Reinhard, and C. Toepffer, Phys. Rev. E 56, 7310 (1997).

${ }^{26}$ J. P. Hansen and I. R. McDonald, Theory of Simple Liquids (Academic, London, 1986).

${ }^{27}$ Y. Rosenfeld, Phys. Rev. A 15, 2545 (1977).

${ }^{28}$ Y. Rosenfeld, J. Phys. IV 10, Pr5-129 (2000).

${ }^{29}$ F. A. Lindemann, Z. Phys. 11, 609 (1910).

${ }^{30}$ J. J. Erpenbeck, Phys. Rev. A 38, 6255 (1988).

${ }^{31}$ H. Totsuji, H. Shirokoshi, and S. Nara, Phys. Lett. A 162, 174 (1992).

${ }^{32}$ W. H. Press, S. A. Teukolsky, W. T. Vetterling, and B. P. Flannery, Numerical Recipes in C (Cambridge University Press, Cambridge, 1988), Chap. 12.

${ }^{33}$ S. Hamaguchi and H. Ohta, J. Phys. IV 10, Pr5-17 (2000).

${ }^{34}$ H. Ohta and S. Hamaguchi, Phys. Rev. Lett. 84, 6026 (2000). 